\title{
RF Loss Model for Tree Canopies with Varying Water Content
}

\author{
Sonam Peden ${ }^{1,2 *}$, Ronald C. Bradbury ${ }^{1}$, David William Lamb ${ }^{1,3}$, Mark Hedley ${ }^{4}$ \\ ${ }^{1}$ Precision Agriculture Research Group, University of New England, Armidale, NSW, Australia \\ ${ }^{2}$ College of Science and Technology, Royal University of Bhutan, Rinchending, Bhutan \\ ${ }^{3}$ Food Agility Cooperative Research Centre, University of New England, Armidale, NSW, Australia \\ ${ }^{4}$ CSIRO Data61, Marsfield, NSW, Australia \\ Email: *speden@myune.edu.au
}

How to cite this paper: Peden, S., Bradbury, R.C., Lamb, D.W. and Hedley, M. (2021) RF Loss Model for Tree Canopies with Varying Water Content. Journal of Electromagnetic Analysis and Applications, 13, 83-101.

https://doi.org/10.4236/jemaa.2021.136006

Received: June 3, 2021

Accepted: June 27, 2021

Published: June 30, 2021

Copyright $\odot 2021$ by author(s) and Scientific Research Publishing Inc. This work is licensed under the Creative Commons Attribution International License (CC BY 4.0).

http://creativecommons.org/licenses/by/4.0/

\section{Open Access}

\begin{abstract}
Detection of plant water status is important for monitoring plant physiology. Previous studies showed that radio waves are attenuated when passing through vegetation such as trees, and models (both empirical and analytical) were developed. However, for models to be more broadly applicable across a broad range of vegetation types and constructs, basic electrical properties of the vegetation need to be characterised. In our previous work, a model was developed to calculate the RF loss through vegetation with varying water content. In this paper, the model was extended to calculate RF loss through tree canopies with or without an air gap. When the model was compared with the actual RF loss acquired using Eucalyptus blakelyi trees (with and without leaves), there was a systematic offset equivalent to a residual moisture content of $13 \%$ that was attributed to bound water. When the model was adjusted for the additional water content, the effective water path (EWP) was found to explain $72 \%$ of the variance in the measured RF loss.
\end{abstract}

\section{Keywords}

Radio Attenuation, Water Content, Vegetation Thickness, Permittivity, Path Loss

\section{Introduction}

Eucalypts are iconic Australian forest trees. The Eucalyptus forest type is by far the most common forest type in Australia covering 101 million hectares, which is $77 \%$ of Australia's total native forest area [1]. The prolonged drought experienced in southern Australia between 1996 and 2010 (the Millennium Drought) 
caused widespread mortality and secondary insect attack in both eucalypt native forests and pine plantations [2]. Species composition has also changed in response to prolonged lower rainfall [3]. Since the mid-1990s, mainland southeast Australia has experienced an 11 percent reduction in April-October rainfall. Drought makes vegetation more flammable, and therefore more likely to support extreme bushfire behaviour [4].

Water stress affects plant growth and development due to reduction in photosynthetic activities [5] [6] [7] and hence affects forest productivity [8] [9]. Canopy leaf wilting is considered an important visible symptom of drought, when water loss by transpiration is greater than absorption by the roots [10] [11]. The detection of plant water status is important for monitoring the physiological status of plants, and the assessment of drought and fire risk in natural plant communities, and the irrigation scheduling of crops [12] [13]. Although field sampling of single leaves and shoots provides the most accurate assessment of plant water status, such methods are not feasible when estimates are required for large areas of vegetation [14].

Radio signals are attenuated when passing through vegetation due to absorption and scattering by the discrete elements such as the branches, stems and leaves [15] [16] [17]. The so-called RF loss has been measured for specific frequencies in particular situations (forest, apple orchard \& coconut garden for example) and empirical models have been developed from such measurements [16] [18] [19] [20] [21] [22]. Analytical models have also been developed but the more accurate Radiative Energy Transfer (RET) models depend on experimental measurements for their formulation and validation [23]. For a model to be accurate across a broad range of vegetation however, the relevant electrical characteristics of the vegetation need to be incorporated into the model.

Radio waves interact strongly with water [24] and eucalyptus trees are no exception. While RF loss measurements at any radio frequency would be related to water content, some frequency bands are more suitable than others. Below about $600 \mathrm{MHz}$, the main RF loss mechanism involves the movement of ions. RF loss then is highly dependent on the medium's electrical conductivity and hence the concentration of dissolved ions. Such information would be difficult to obtain for different species of vegetation which presents a hurdle for practical estimation of water content. Above about $1 \mathrm{GHz}$ and up to $100 \mathrm{GHz}$, the main RF loss mechanism is the rotation of water molecules resulting from interaction between the radio signal electric field and the molecular electric dipole [25] [26]. Frequencies above $1 \mathrm{GHz}$ have an advantage in that electrical conductivity does not play a significant part hence avoiding the need to characterize the highly-variable constituents of electric conductivity in leaves. The higher frequency also offers another advantage; namely directional antennas that can be used to facilitate location-specific measurements are smaller.

Le Vine and Karam [27] calculated the attenuation associated with a vegetation canopy using a discrete scatter model, where the vegetation canopy is pre- 
sented by a sparse layer of discrete, randomly oriented particles such as leaves, stalks, branches, etc over a homogeneous ground plane (soil). They found that for frequencies up to $5 \mathrm{GHz}$ the attenuation varies approximately linearly with plant water content over the range 0.2 to 0.5 (by volume). Nakajima, et al. [28] measured the RF attenuation of individual leaves at 5,10 and $20 \mathrm{GHz}$ in a waveguide. They also investigated the effect in a living tree by measuring RF attenuation at $10.5 \mathrm{GHz}$. They asserted that "Microwave attenuation by tree foliage should have a strong link to water content in the leaves". The dependency of attenuation on water content is through the dielectric constant which itself is highly dependent on the water content inside the material.

The water inside vegetation (leaves and stems) can be divided into free water and bound water. Free water is the liquid water found in cell lumen and is relatively easy to remove [29]. Bound water is the water molecules that penetrate the cell walls and are chemically bound to cellulose molecules. Bound water cannot always be expelled by heat without damaging the material [30] and the removal of bound water also depends on the temperature and relative humidity of the environment [31].

Previous studies showed that the RF loss through vegetation is strongly dependent on the water content in vegetation through its dielectric constant. Ulaby and El-Rayes [32] describe the relationship between water content and dielectric constant, detailed later in this paper. The relationship between RF loss and water content, however, has not been quantified.

In our previous paper, we developed a model to calculate the RF loss through packed Eucalyptus leaves [33]. In this work, we extend our previous model to calculate the radio frequency (RF) signal loss through tree canopies (combination of vegetation and air) and the model was compared against experimental measurements of RF loss for Eucalyptus tree canopies at $2.4 \mathrm{GHz}$. The key things are 1) to derive a model relating RF loss and water content; 2) using cut trees so that water content could be found by weighing; 3) air-drying to vary the water content while keeping the structure of the tree intact; 4) done inside to reduce environmental changes (rain, wind, temperature) that might otherwise affect measurements.

\section{Model}

In the following model derivation, the radio wave electric field is first related to the intrinsic impedance and complex propagation constant of each medium in the path. Those two parameters are then related to the corresponding complex permittivity of each medium. Permittivity, in turn, is related further to the volume fractions of the substances (especially water) within the media.

\subsection{Wave Propagation}

We assume a plane wave travels through a set of slabs. In the case of a packed canopy (a group of trees with no space in between), each slab in the model 
represents one tree. For an open canopy on the other hand, each tree and each space between the trees is represented by a slab in the model (refer Figure 1).

Each tree is assumed to be a homogenous, lossy medium. Also, we assume that the material is non-magnetic.

In Figure 2, we suppose that a radio wave is emitted from a transmitting antenna on the left, travels from left-to-right through the media and proceeds to a receiving antenna on the right. We are interested in the RF loss in intensity above the free-space loss. The model considers three components to the RF loss: 1) partial transmission at the interfaces; 2) partial reflection at the interfaces and 3) absorption by the lossy media.

When an incident electromagnetic wave with electric field phasor, $E_{T F}$ is incident at the first interface, it is partially transmitted and partially reflected. The transmitted wave, $E_{1 F}$ propagates through first lossy medium with a complex
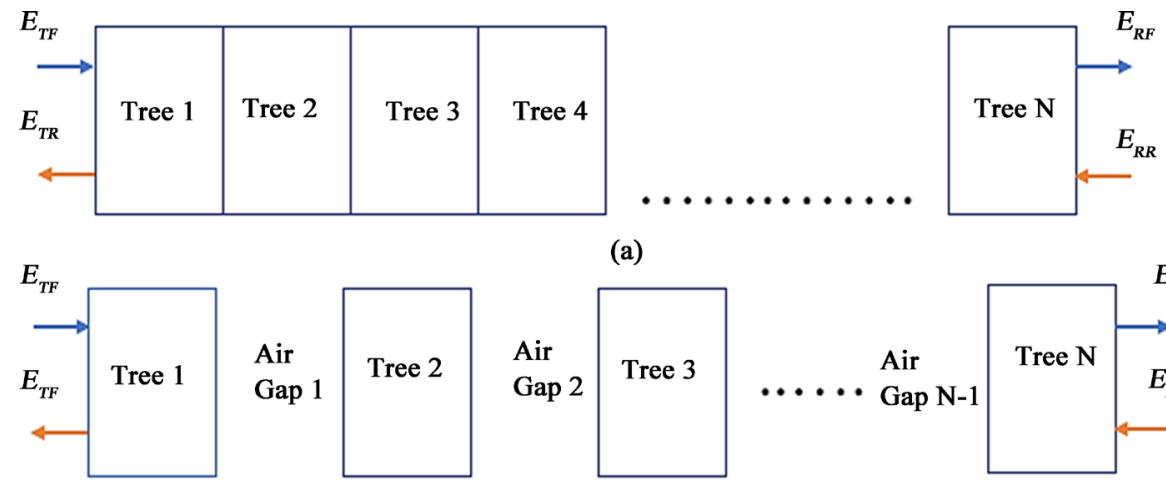

(a)

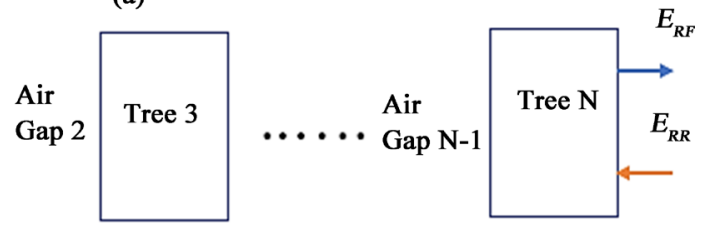

(b)

Figure 1. Model when transmitted wave propagates through (a) $N$ number of trees placed in series without an air gap in between the trees and (b) $N$ number of trees placed in series with $N$-1 air gaps in between the trees.

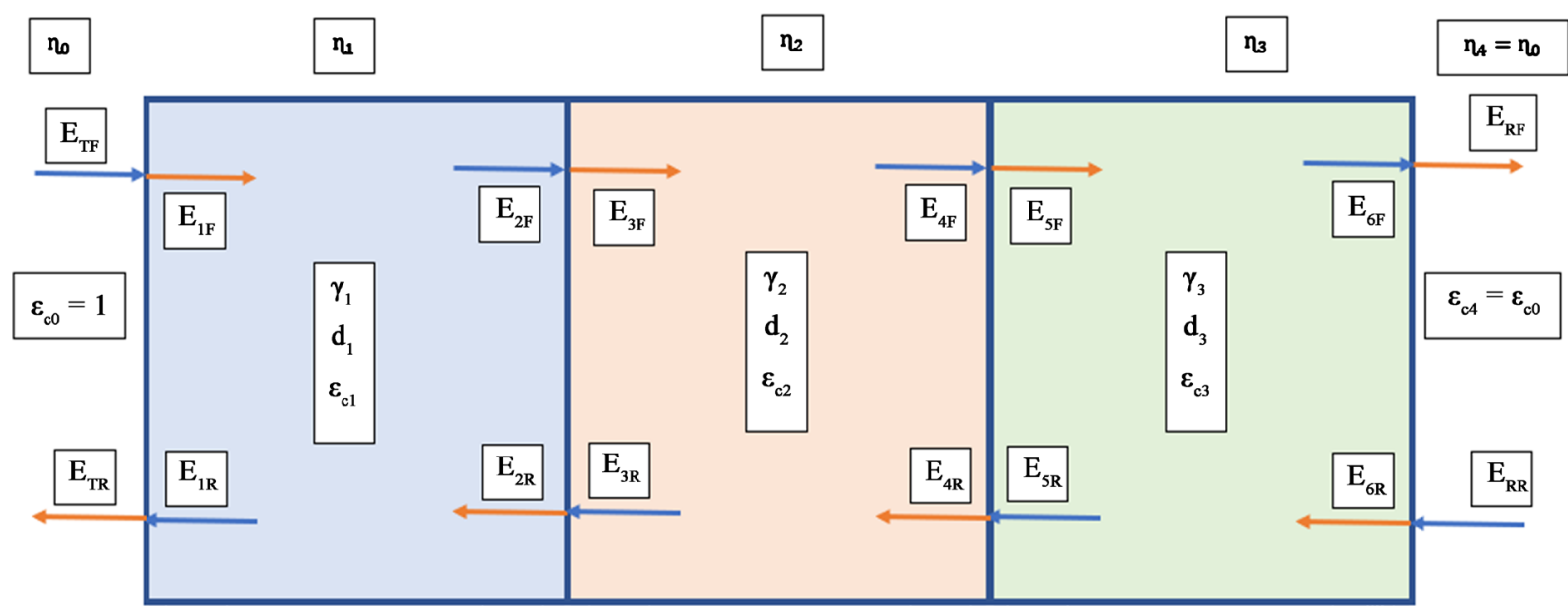

Figure 2. Structure of 3 lossy medium slabs and 4 interfaces. Symbols for the electromagnetic properties of the materials are defined. When a plane radio wave travelling left-to-right meets the medium it is partially transmitted and partially reflected at the first interface. The transmitted wave propagates through the lossy medium with a complex propagation constant $\gamma_{1}$. At the second interface it is again partially transmitted and partially reflected, the transmitted wave propagates through second medium and so on until it transmits back in to the air. 
propagation $\gamma_{1}$, intrinsic impedance $\eta_{1}$ and thickness $d_{1}$ in metres. At the second interface it is again partially transmitted and partially reflected, the transmitted wave again passes through second lossy medium and so on until it transmits through the last interface into the air as $E_{R F}$. As a result of the reflections there is a reverse-travelling wave also, denoted with subscript $R$.

In Figure 2, $\gamma_{1}, \gamma_{2}, \gamma_{3}$ are the complex propagation constants in the 3 different media with thicknesses $d_{1}, d_{2}, d_{3}$, intrinsic impedances $\eta_{1}, \eta_{2}, \eta_{3}$ and complex permittivity $\varepsilon_{c 1}, \varepsilon_{c 2}, \varepsilon_{c 3}$ respectively.

We consider Figure 2 to be cascaded two-port networks and the number of two-port networks with their terminals according to Figure 2 are shown in Figure 3.

\subsubsection{First Two-Port Network}

The combined effect of cascaded two-port networks is found by multiplying the individual $\mathrm{T}$-parameter matrices, but $\mathrm{S}$-parameters are more intuitive. The $\mathrm{S}$-parameter matrix equation is found first and then converted to a T-parameter equation.

The first two-port network represents the interaction of the radio waves at the left-most interface in Figure 2. The incoming phasors, $E_{T F}$ and $E_{1 R}$, are related to the outgoing phasors, $E_{T R}$ and $E_{1 P}$ by an S-parameter matrix [34] as

$$
\left[\begin{array}{c}
E_{T R} \\
E_{1 F}
\end{array}\right]=\left[\begin{array}{cc}
\Gamma_{01} & t_{10} \\
t_{01} & \Gamma_{10}
\end{array}\right]\left[\begin{array}{c}
E_{T F} \\
E_{1 R}
\end{array}\right],
$$

where, $t_{01}$ and $t_{10}$ are the transmission coefficients of a forward and reverse travelling waves through the first interface respectively, $\Gamma_{10}$ and $\Gamma_{01}$ are the reverse-to-forward and forward-to-reverse reflection coefficients when waves are reflected from the first interface (air-to-first lossy medium interface). The transmission coefficients, $t_{01}$ and $t_{10}$ are complex and these represent both the amplitude change and the radio signal phase shift that occurs when the wave passes through the interfaces. Likewise, the reflection coefficients, $\Gamma_{10}$ and $\Gamma_{01}$ are complex and represent the amplitude and phase shift from reflection. These terms are expressed in the following Equations (2)-(5) [35]:

$$
\begin{gathered}
\Gamma_{10}=\frac{\eta_{0}-\eta_{1}}{\eta_{0}+\eta_{1}}=\frac{\sqrt{\varepsilon_{c 1}}-\sqrt{\varepsilon_{c 0}}}{\sqrt{\varepsilon_{c 1}}+\sqrt{\varepsilon_{c 0}}}, \\
\Gamma_{01}=\frac{\eta_{1}-\eta_{0}}{\eta_{0}+\eta_{1}}=-\left(\frac{\sqrt{\varepsilon_{c 1}}-\sqrt{\varepsilon_{c 0}}}{\sqrt{\varepsilon_{c 1}}+\sqrt{\varepsilon_{c 0}}}\right),
\end{gathered}
$$

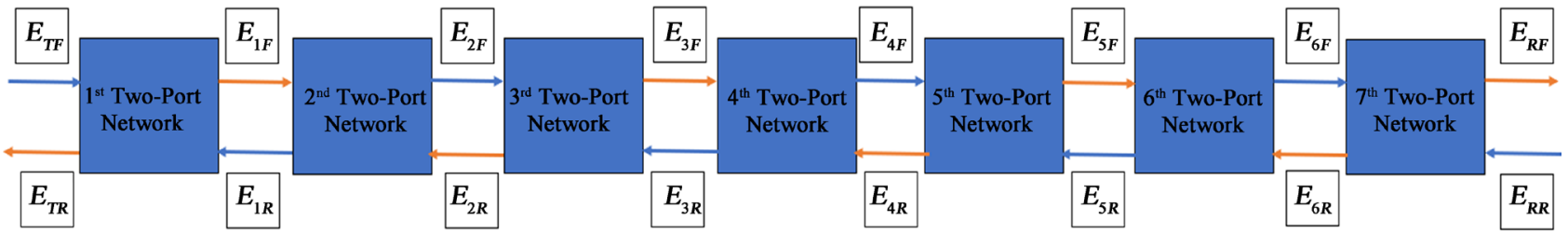

Figure 3. Cascade connection of Two-port networks for the model shown in Figure 2. 


$$
\begin{aligned}
& t_{01}=\frac{2 \eta_{1}}{\eta_{0}+\eta_{1}}=\frac{2 \sqrt{\varepsilon_{c 0}}}{\sqrt{\varepsilon_{c 1}}+\sqrt{\varepsilon_{c 0}}}, \\
& t_{10}=\frac{2 \eta_{0}}{\eta_{0}+\eta_{1}}=\frac{2 \sqrt{\varepsilon_{c 1}}}{\sqrt{\varepsilon_{c 1}}+\sqrt{\varepsilon_{c 0}}},
\end{aligned}
$$

where, $\eta_{0}$ and $\eta_{1}$ are the intrinsic impedances of air and the first lossy medium respectively, and $\varepsilon_{c 0}$ is the relative permittivity of air $(=1)$.

Converting the S-parameter matrix to T-parameter matrix [34], Equation (1) can be written as

$$
\left[\begin{array}{c}
E_{T R} \\
E_{T F}
\end{array}\right]=\left[\begin{array}{cc}
\frac{1}{t_{01}} & \frac{\Gamma_{01}}{t_{01}} \\
\frac{-\Gamma_{10}}{t_{01}} & \frac{1}{t_{01}}
\end{array}\right]\left[\begin{array}{l}
E_{1 R} \\
E_{1 F}
\end{array}\right] .
$$

\subsubsection{Second Two-Port Network}

The output electric field phasor, $E_{1 F}$ of first two-port network travels through the first lossy medium and comes out of the medium attenuated as $E_{2 F}$ Similarly, $E_{2 R}$ enters the lossy medium and exits attenuated as $E_{1 R}$. The S-parameter for this two-port network can be written as

$$
\left[\begin{array}{c}
E_{1 R} \\
E_{2 F}
\end{array}\right]=\left[\begin{array}{cc}
0 & \mathrm{e}^{-\gamma_{1} d_{1}} \\
\mathrm{e}^{-\gamma_{1} d_{1}} & 0
\end{array}\right]\left[\begin{array}{c}
E_{1 F} \\
E_{2 R}
\end{array}\right],
$$

where, $\gamma_{1}$ is the complex propagation constant of the first lossy medium. Complex propagation constant of a sinusoidal electromagnetic wave is a measure of the change undergone by the amplitude and phase of the wave as it propagates in a given direction. The real part of $\gamma$ is the attenuation constant, $\alpha$ in Np/m (Nepers per $\mathrm{m}$ ) and the imaginary part is the phase constant, $\beta$ in $\mathrm{rad} / \mathrm{m}$ [35]. The complex propagation constant can be expressed as [35]:

$$
\gamma_{1}=j \omega \sqrt{\mu_{0} \varepsilon_{0} \varepsilon_{c 1}},
$$

where, $\varepsilon_{0}$ and $\mu_{0}$ are the permittivity and permeability of vacuum respectively, $\omega$ is the angular frequency in $\mathrm{rad} / \mathrm{sec}$ and $\varepsilon_{c 1}$ is the complex permittivity of the first lossy medium. Complex permittivity, $\varepsilon_{c 1}$ is expressed as

$$
\varepsilon_{c 1}=\varepsilon_{c 1}^{\prime}-j \varepsilon_{c 1}^{\prime \prime},
$$

where, the real part, $\varepsilon_{c 1}^{\prime}$ represents the relative permittivity and the imaginary part, $\varepsilon_{c 1}^{\prime \prime}$ represents the dielectric loss [30].

Converting the S-parameter matrix to T-parameter matrix, Equation (7) can be written as

$$
\left[\begin{array}{c}
E_{1 R} \\
E_{1 F}
\end{array}\right]=\left[\begin{array}{cc}
\mathrm{e}^{-\gamma_{1} d_{1}} & 0 \\
0 & \mathrm{e}^{\gamma_{1} d_{1}}
\end{array}\right]\left[\begin{array}{c}
E_{2 R} \\
E_{2 F}
\end{array}\right] .
$$

The output electric field phasor, $E_{2 F}$ of second two-port network transmits through the second interface, travels through the second lossy medium and it continues until it transmits into the air through the last interface, which is the 
seventh two-port network as per Figure 3.

The electric field phasor on the left-hand side of the cascaded 7 two-port networks can be written as simple multiplication of the T-parameter matrices and the electric field phasor on the right-hand side as

$$
\begin{aligned}
{\left[\begin{array}{l}
E_{T R} \\
E_{T F}
\end{array}\right]=} & {\left[\begin{array}{cc}
\frac{1}{t_{01}} & \frac{\Gamma_{01}}{t_{01}} \\
\frac{-\Gamma_{10}}{t_{01}} & \frac{1}{t_{01}}
\end{array}\right]\left[\begin{array}{cc}
\mathrm{e}^{-\gamma_{1} d_{1}} & 0 \\
0 & \mathrm{e}^{\gamma_{1} d_{1}}
\end{array}\right]\left[\begin{array}{cc}
\frac{1}{t_{12}} & \frac{\Gamma_{12}}{t_{12}} \\
\frac{-\Gamma_{21}}{t_{12}} & \frac{1}{t_{12}}
\end{array}\right]\left[\begin{array}{cc}
\mathrm{e}^{-\gamma_{2} d_{2}} & 0 \\
0 & \mathrm{e}^{\gamma_{2} d_{2}}
\end{array}\right] } \\
& \times\left[\begin{array}{cc}
\frac{1}{t_{23}} & \frac{\Gamma_{23}}{t_{23}} \\
\frac{-\Gamma_{32}}{t_{23}} & \frac{1}{t_{23}}
\end{array}\right]\left[\begin{array}{cc}
\mathrm{e}^{-\gamma_{3} d_{3}} & 0 \\
0 & \mathrm{e}^{\gamma_{3} d_{3}}
\end{array}\right]\left[\begin{array}{cc}
\frac{1}{t_{34}} & \frac{\Gamma_{34}}{t_{34}} \\
\frac{-\Gamma_{43}}{t_{34}} & \frac{1}{t_{34}}
\end{array}\right]\left[\begin{array}{l}
E_{R R} \\
E_{R F}
\end{array}\right]
\end{aligned}
$$

where the incident wave at the rightmost interface $E_{R R}=0$. If there are $N$ number of lossy media then Equation (11) can be written as

$$
\begin{aligned}
{\left[\begin{array}{c}
E_{T R} / E_{R F} \\
E_{T F} / E_{R F}
\end{array}\right]=} & {\left[\begin{array}{cc}
\frac{1}{t_{01}} & \frac{\Gamma_{01}}{t_{01}} \\
\frac{-\Gamma_{10}}{t_{01}} & \frac{1}{t_{01}}
\end{array}\right]\left[\begin{array}{cc}
\mathrm{e}^{-\gamma_{1} d_{1}} & 0 \\
0 & \mathrm{e}^{\gamma_{1} d_{1}}
\end{array}\right] \cdots\left[\begin{array}{cc}
\frac{1}{t_{N-1, N}} & \frac{\Gamma_{N-1, N}}{t_{N-1, N}} \\
\frac{-\Gamma_{N, N-1}}{t_{N-1, N}} & \frac{1}{t_{N-1, N}}
\end{array}\right] } \\
& \times\left[\begin{array}{cc}
\mathrm{e}^{-\gamma_{N} d_{N}} & 0 \\
0 & \mathrm{e}^{\gamma_{N} d_{N}}
\end{array}\right]\left[\begin{array}{cc}
\frac{1}{t_{N, N+1}} & \frac{\Gamma_{N, N+1}}{t_{N, N+1}} \\
\frac{-\Gamma_{N+1, N}}{t_{N, N+1}} & \frac{1}{t_{N, N+1}}
\end{array}\right]\left[\begin{array}{l}
0 \\
1
\end{array}\right]
\end{aligned}
$$

Then the total loss for Nlossy homogenous slabs in $\mathrm{dB}$ is

$$
L_{\text {slab }}=20 \log _{10}\left|\frac{E_{T F}}{E_{R F}}\right| \text {. }
$$

Equation (12) in this paper simplifies to Equation (7) in Peden, et al. [33], in the special case of $N=1$ medium.

\subsection{Complex Permittivity of a Tree Canopy}

If the lossy homogenous medium mentioned in Section 2.1 is a tree canopy, then the permittivity $\mathcal{E}_{c}$ in Equation (8) and (9) is the permittivity of a tree canopy. A canopy of a tree consists of leaves, branches/twigs and air, and all these will contribute to the total permittivity of a tree canopy given by,

$$
\varepsilon_{c}=v_{v} \varepsilon_{v}+v_{a} \varepsilon_{a}
$$

where, $\varepsilon_{v}$ and $\varepsilon_{a}$ are the permittivity of the vegetation (leaves and branches/twigs) and air in the canopy respectively. $v_{V}$ and $V_{a}$ are the volume fractions of the vegetation and air in the canopy envelope respectively.

Ulaby and El-Rayes [32] developed a dielectric model to calculate the dielectric constant of vegetation. They modelled the dielectric constant of vegetation, $\mathcal{E}_{V}$ as a simple addition of three components: a nondispersive residual component 
$\left(\varepsilon_{r}\right)$, free-water component $\left(V_{f w} \varepsilon_{f}\right)$ and the bulk vegetation-bound water component $\left(v_{b} \varepsilon_{b}\right)$, expressed as

$$
\varepsilon_{v}=\varepsilon_{r}+v_{f w} \varepsilon_{f}+v_{b} \varepsilon_{b},
$$

where, $v_{f w}$ is the volume fraction of free water, $\varepsilon_{f}$ is the dielectric constant of free water, $v_{b}$ is the volume fraction of the bulk vegetation-bound water mixture and $\varepsilon_{b}$ is its dielectric constant. Assuming that $\varepsilon_{r}$ is a nondispersive residual component is supported by Hasted [30] who states that the dielectric loss of many dry materials is low in the microwave band, having values between $10^{-1}$ and $10^{-3}$.

Free water may contain dissolved salt and the frequency dependent dielectric constant of bulk saline water is given by the Debye equation [36],

$$
\varepsilon_{f}=\varepsilon_{f}^{\prime}-j \varepsilon_{f}^{\prime \prime}=\varepsilon_{f \infty}+\frac{\varepsilon_{f s}-\varepsilon_{f \infty}}{1+j \frac{f}{f_{f 0}}}-j \frac{\sigma}{2 \pi f \varepsilon_{0}},
$$

where, $f$ is the operating frequency in $\mathrm{Hz}, f_{00}$ is the relaxation frequency in $\mathrm{Hz}$, and $\varepsilon_{f s}$ and $\varepsilon_{f o}$ are the dimensionless static and high frequency limits of $\varepsilon_{f}^{\prime}$. The salinity, $S$ of a solution is defined as the total mass of salt in grams dissolved in a solution of $1 \mathrm{~kg}$ and is expressed as parts per thousand on a weight basis. The salinity for vegetation is taken to be $10 \%$ [37]. For salinity, $S \leq 10 \%$ and at room temperature, Equation (16) could be approximated as,

$$
\varepsilon_{f}=4.9+\frac{75}{1+j \frac{f}{18}}-j \frac{\sigma 18}{f},
$$

where, $f$ is in GHz. The conductivity $\sigma$ (siemen/metre) may be related to $S(\%)$ by,

$$
\sigma \cong 0.16 S-0.0013 S^{2}
$$

For bound water, Ulaby and El-Rayes [32] conducted dielectric measurements on sucrose-water mixture and data was fitted to Cole-Cole dispersion equation. The complex dielectric constant of bound water is given by

$$
\varepsilon_{b}=2.9+\frac{55}{1+\left(\frac{j f}{0.18}\right)^{0.5}},
$$

where, $f$ is in GHz. Equation (17) includes a loss term associated with the conductivity of the free water and dissolved ions in the medium. In contrast, Equation (19) has no corresponding conductivity term as the water molecules are bound to other substances and do not contribute to bulk conductivity of the medium.

By inserting Equations (17) and (19) in Equation (15), the dielectric constant of a vegetation can be written as

$$
\varepsilon_{v}=\varepsilon_{r}+v_{f w}\left(4.9+\frac{75}{1+j \frac{f}{18}}-j \frac{\sigma 18}{f}\right)+v_{b}\left(2.9+\frac{55}{1+\left(\frac{j f}{0.18}\right)^{0.5}}\right) .
$$


The variation of $\varepsilon_{r}, V_{f w}$ and $v_{b}$ with gravimetric moisture content, $M_{g}$ were derived by Ulaby and El-Rayes [32] by fitting their model (Equation (20)) to complex permittivity measurements acquired using corn leaves and verified against corn stalks, soybean leaves, aspen leaves, balsam fir trunk, potatoes, apples, and other types of vegetation material. The empirical equations are as follows:

$$
\begin{gathered}
\varepsilon_{r}=1.7-0.74 M_{g}+6.16 M_{g}^{2}, \\
v_{f w}=M_{g}\left(0.55 M_{g}-0.076\right), \\
v_{b}=\frac{4.64 M_{g}^{2}}{1+7.36 M_{g}^{2}},
\end{gathered}
$$

where, $M_{g}$ is calculated from the weight measurement of tree before and after drying as follows

$$
M_{g}=1-\left(\frac{\text { weight of dry tree }}{\text { weight of tree (different stages of drying })}\right) .
$$

By inserting Equation (20) in Equation (14), the dielectric constant of a tree canopy can be written as

$\varepsilon_{c}=\left[\left\{\varepsilon_{r}+v_{f w}\left(4.9+\frac{75}{1+j \frac{f}{18}}-j \frac{\sigma 18}{f}\right)+v_{b}\left(2.9+\frac{55}{1+\left(\frac{j f}{0.18}\right)^{0.5}}\right)\right\} \times v_{v}\right]+v_{a} \varepsilon_{a}$.

\section{Material and Method}

\subsection{Experimental Site and Equipment Used}

All the experiments were conducted in an indoor facility at The University of New England main campus located in Armidale, New South Wales, Australia. Two flat-panel, phased-array directional antennas (ARC Wireless Solutions, USA, PA2419B01, $39.1 \mathrm{~cm} \times 39.1 \mathrm{~cm} \times 4.3 \mathrm{~cm}$ ) were used, one as a transmitter connected to a transceiver Beacon (Dosec Design, Australia, EnviroNode Beacon) and the other as a receiver connected to a transceiver hub (Dosec Design, Australia, EnviroNode Hub) operated at a frequency of $2.4331 \mathrm{GHz}$. The antenna had a gain of $19 \mathrm{dBi}$, front-to-back ratio of $>30 \mathrm{~dB}$ and $3 \mathrm{~dB}$ beamwidth of $\pm 9^{\circ}$. The antennas were placed facing each other at a separation of 6.15 metres. A constant transmitted power of 100 milliwatts was used. The hub measured and logged the RSSI (received signal strength indicator, $\mathrm{dBm}$ ) to a removable SD card at 1-minute intervals. The experimental set-up is shown in Figure 4.

A Eucalyptus blakelyi (also known as Blakely's red gum) tree about $2.6 \mathrm{~m}$ in height (Tree 1) was cut and was mounted on a wooden pallet. The RSSI (dBm) for no obstruction between the transceivers was measured for 4 minutes and then the tree was placed in front of one antenna. The difference between the time-average RSSI with and without the tree in place was converted to a time-averaged RF loss associated with the tree. The sequence of tree and no tree 


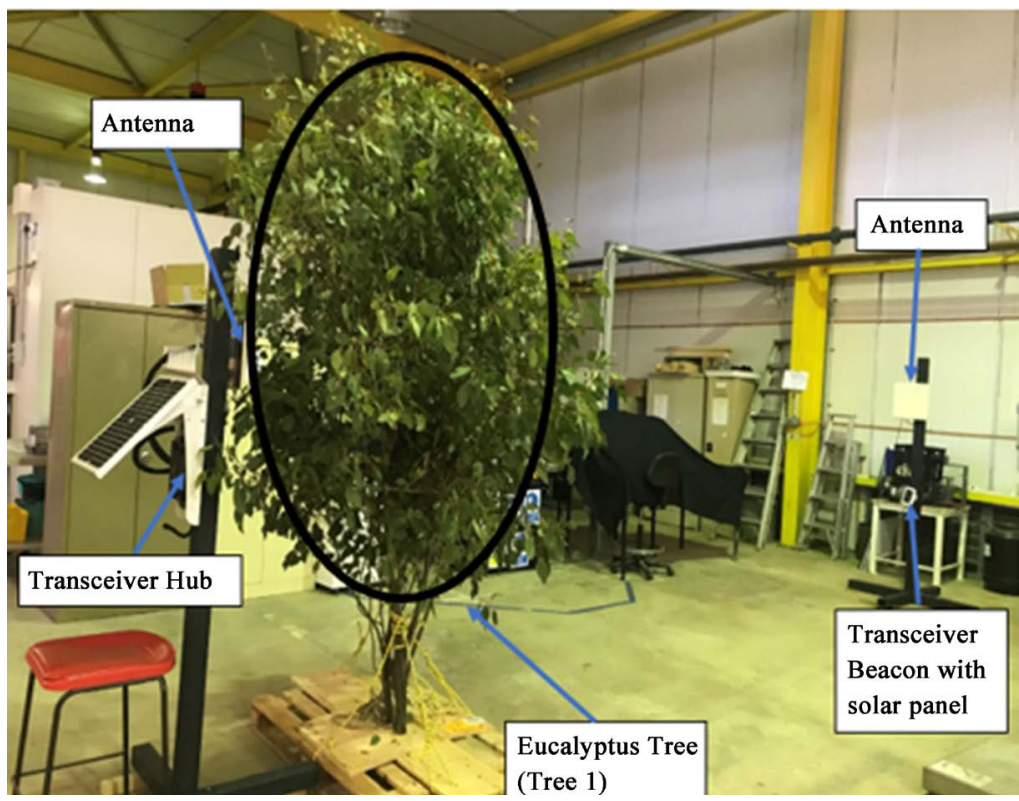

Figure 4. Experimental set-up within the indoor facility. Two flat-panel antennas were mounted facing each other $6.15 \mathrm{~m}$ apart. A tree was placed immediately in front of one antenna. The RSSI $(\mathrm{dBm})$ was recorded to a removable SD card inside the transceiver Hub every minute. The tree canopy can be approximated as an ellipse (black annotation).

measurements was repeated three times to provide a measurement average. The $\mathrm{RF}$ loss $(L)$ associated with the tree canopy was then calculated using,

$$
L(\mathrm{~dB})=\operatorname{RSSI}(\text { no tree })-\operatorname{RSSI}(\text { tree }) .
$$

Following the RSSI measurements with and without the tree in place, the tree was left to dry for one hour and the measurement RF loss was repeated.

The process of drying and remeasuring the RSSI was repeated until no further weight loss from drying was achieved (i.e. tree was considered dry). At this end point the mass of the water $\left(m_{w}\right)$ in the tree canopy and subsequently each partially-dried tree canopy was retrospectively calculated from the known mass of the tree during drying and the final dry weight of the tree.

The measurement sequence was repeated for another tree with leaves (Tree 2) and a third, bare tree without leaves (Bare tree) as shown in Figure 5. Note that the bare tree was left to dry for a day rather than an hour before the measurement was repeated. The measurement sequence was also repeated for two trees positioned in series as shown in Figure 6.

\subsection{Calculation of Volume Fractions}

The tree canopy was considered as an ellipsoid (refer Figure 4) and the volume of the tree canopy envelope was calculated as follows

$$
V=\frac{\pi}{6} \times A \times B \times C,
$$

where, $A, B$ and $C$ are the lengths of the principal axes and these lengths were measured using a measuring tape for each tree. $A, B$ and $C$ of the trees used for 
the experiments are listed in Table 1.

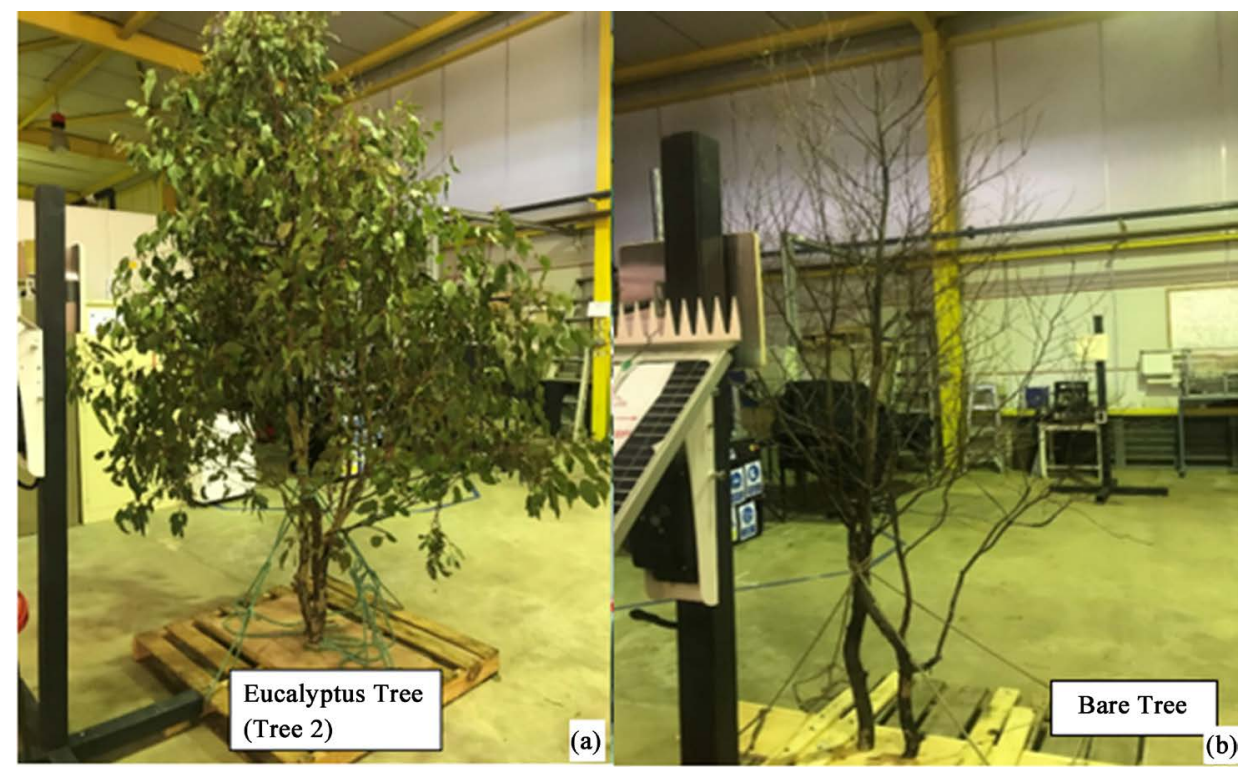

Figure 5. (a) Tree 2 with leaves and (b) Bare tree positioned in between the transceivers.

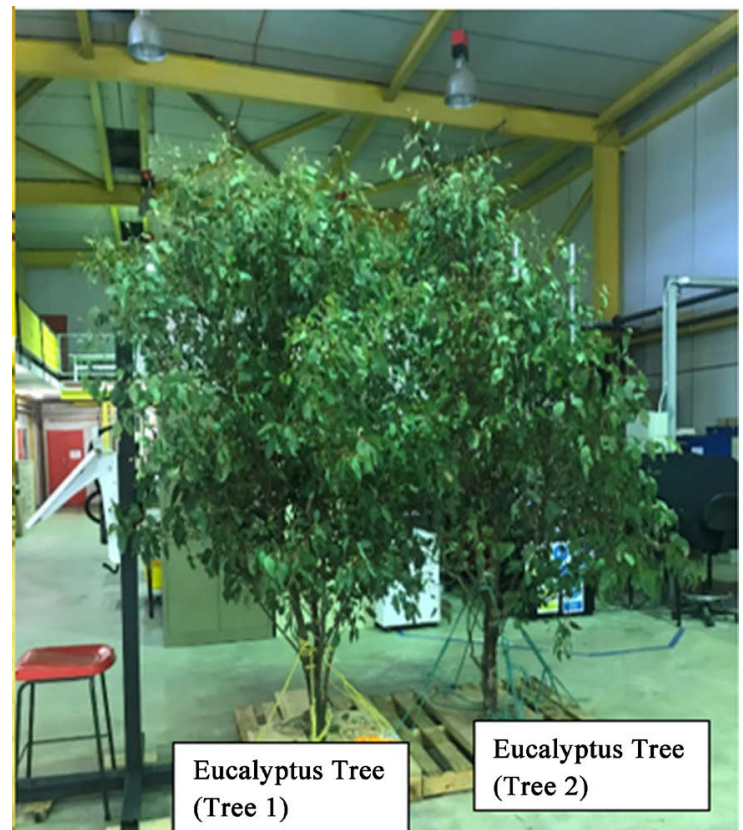

Figure 6. Two trees (Tree 1, Tree 2) positioned in series in between the transceivers.

Table 1. Dimensions of a tree to calculate the volume of the tree. The tree canopy was considered as an ellipsoid and $A, B$ and $C$ are the three lengths of the principle axes of an ellipsoid in metres.

\begin{tabular}{ccccc}
\hline & Eucalyptus Tree & $\boldsymbol{A}(\mathrm{m})$ & $\boldsymbol{B}(\mathrm{m})$ & $\boldsymbol{C}(\mathrm{m})$ \\
\hline 1 & Tree 1 with leaves & 1.30 & 1.70 & 2.10 \\
2 & Tree 2 with leaves & 1.55 & 1.80 & 2.15 \\
3 & Bare tree & 1.36 & 1.40 & 2.10 \\
\hline
\end{tabular}


The volume fraction of vegetation, $V_{V}$ is a summation of volume fraction of the leaves in the canopy, $V_{L}$ and the volume fraction of the woody part (branches) in the canopy, $V_{\text {wood }}$ are given by,

$$
\begin{gathered}
v_{v}=v_{L}+v_{\text {wood }}, \\
v_{L}=V_{L} / V, \\
v_{\text {wood }}=V_{\text {wood }} / V,
\end{gathered}
$$

where, $V_{L}$ and $V_{\text {wood }}$ are the volume of leaves and woody part of the canopy respectively. Then the volume fraction of air, $V_{a}$ is given by

$$
v_{a}=1-v_{v} .
$$

The volume of leaves and woody part of the canopy are calculated using their mass and density as shown below

$$
\begin{gathered}
V_{L}=m_{L} / \rho_{L}, \\
V_{\text {wood }}=m_{\text {wood }} / \rho_{\text {wood }},
\end{gathered}
$$

where $m_{L}$ and $m_{\text {wood }}$ are the weight of the leaves and woody part in the canopy respectively. The leaves were taken off from the (third) tree and weighed, and the tree was weighed separately in order to yield the values for $m_{L}$ and $m_{\text {wood. }}$. The densities of the leaves, $\rho_{L}=876 \mathrm{~kg} / \mathrm{m}^{3}$ and woody parts, $\rho_{\text {wood }}=1110 \mathrm{~kg} / \mathrm{m}^{3}$ were determined from the measurements of weight and volume of fresh leaves and fresh woody parts respectively. The volume was measured using a displacement method in water. An assumption was made here that the leaves do not shrink when the tree canopy dries out and the volume remains the same throughout the measurement period.

\subsection{Calculation of Effective Water Path (EWP)}

The radio wave passes through a tree with vegetation thickness, $d$ containing a distributed mass, $m_{w}$ of water $(\mathrm{kg})$, we introduce the effective water path (EWP) in mm expressed as

$$
\mathrm{EWP}=\left(\frac{m_{w} \times d}{\rho_{w} \times V}\right) \times 1000,
$$

where, $V$ is the volume of the tree canopy envelope in $\mathrm{m}^{3}$ (refer Equation (27)), $\rho_{w}$ is the density of pure water $\left(1000 \mathrm{~kg} / \mathrm{m}^{3}\right)$ and $d$ in our experiment is equal to the dimension A mentioned in Table 1.

For $N$ number of trees, EWP is a summation of EWP of each tree as follows

$$
\mathrm{EWP}=\mathrm{EWP}_{1}+\mathrm{EWP}_{2}+\mathrm{EWP}_{3}+\cdots+\mathrm{EWP}_{N} .
$$

\section{Results and Discussion}

The measured 2.4 GHz RF loss through the tree canopies for Tree 1, Tree 2, Bare tree (after leaves were removed by hand) and Trees 1 and 2 in series are depicted in Figure 7. The RF loss in $\mathrm{dB}$ is maximum when the leaves are fresh and the RF loss trends down with the reduction of EWP associated with drying. The modelled 

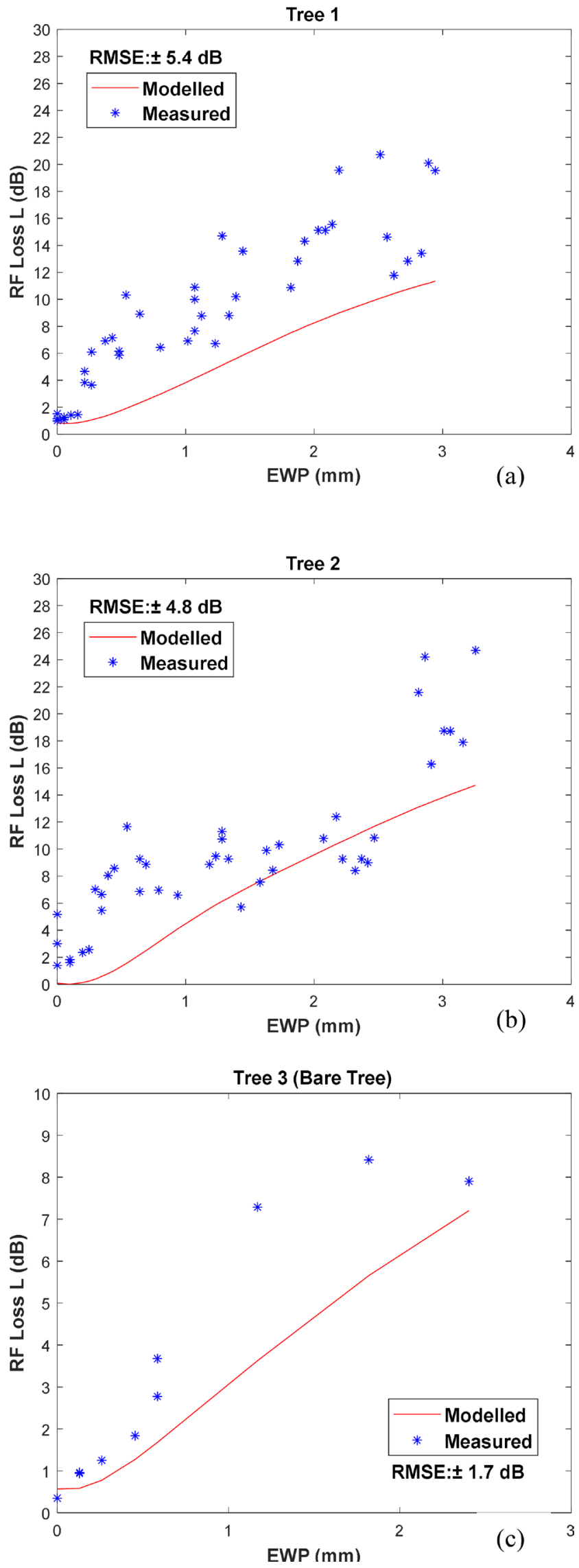


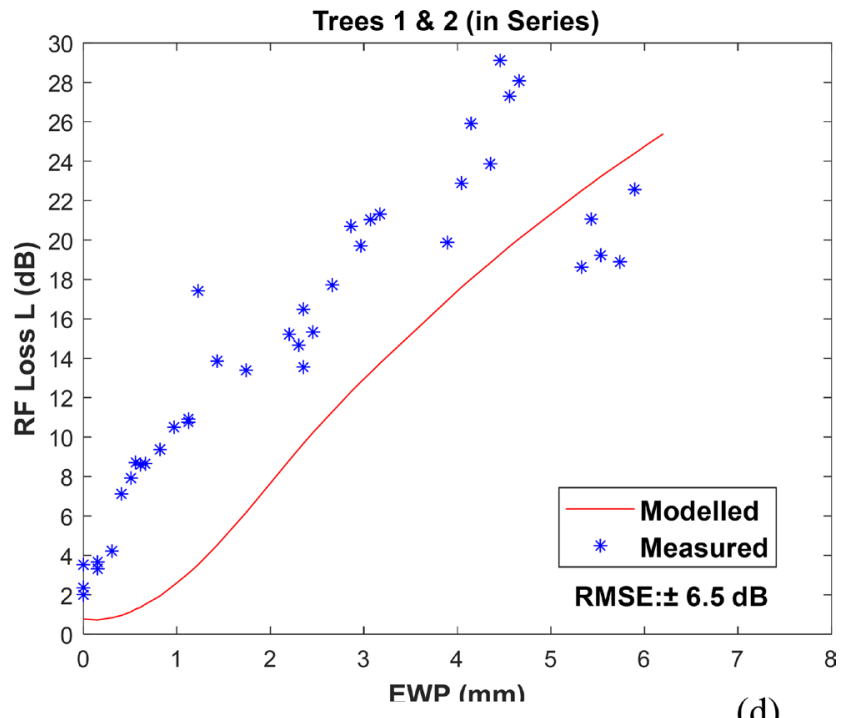

(d)

Figure 7. Plots of measured and modelled (Equation (13)) RF loss (dB) as functions of EWP (mm) for Eucalyptus blakelyi trees ((a) Tree 1; (b) Tree 2; (c) Bare tree; (d) Trees 1 $\& 2$ in series).

values of the RF loss versus EWP (Equation (13)) is also depicted in the graphs of Figure 7 (red curves).

The volume fractions were calculated using the equations mentioned in Section 3.2. The tree canopy consisted of $0.6 \%$ vegetation $\left(v_{v}\right)$ and the remainder is air $\left(v_{a}\right)$. The vegetation $(0.6 \%)$ subdivides to $0.2 \%$ leaves $\left(v_{L}\right)$ and $0.4 \%$ woody parts $\left(v_{\text {wood }}\right)$ of the tree canopy. For the bare tree, $v_{L}=0 \%, v_{\text {wood }}=0.4 \%$ and the remainder is air. An assumption here was made that these volume fractions remain unchanged throughout the experimental period.

The measured RF loss is generally higher than modelled in all the cases shown in Figure 7 irrespective of whether the tree canopy had leaves or not, or whether there was a single tree or two trees in series in between the transceivers. The consistent offset between the measured and modelled values, we believe, is attributable to not adequately accounting for the residual water in estimating EWP using Equation (34). The method we used to dry the leaves may not have removed the water completely, especially the bound water [30] [31]. When dried to a constant weight, vegetation is in an equilibrium state with the drying air [31]. Moreover, the tree may then re-absorb water from the air when the experiment was being carried out.

Quantifying the bound water in leaves, on the other hand, is difficult although it can be estimated using a calorimetric methodology [38] [39] [40]. Note this methodology refers to the notion of unbound and bound water as being, respectively, "freezable" and "unfreezable". Assuming they are related, we were unable to discern this value for eucalyptus leaves using available literature. However, Whitman [41] provides an insight into at least the possible orders of magnitude of this value on the basis of his work on a range of Prairie grasses in the U.S. during the summer season. Sagebrush, for example, has a bound water content 

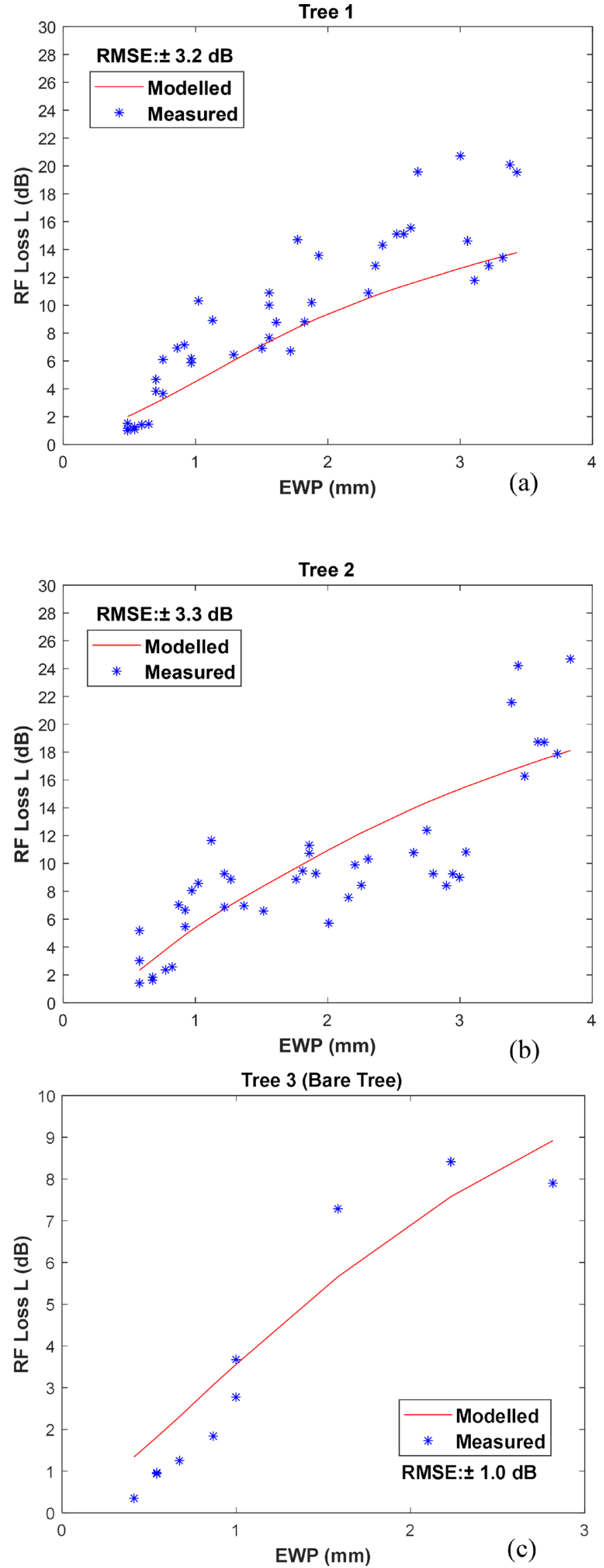


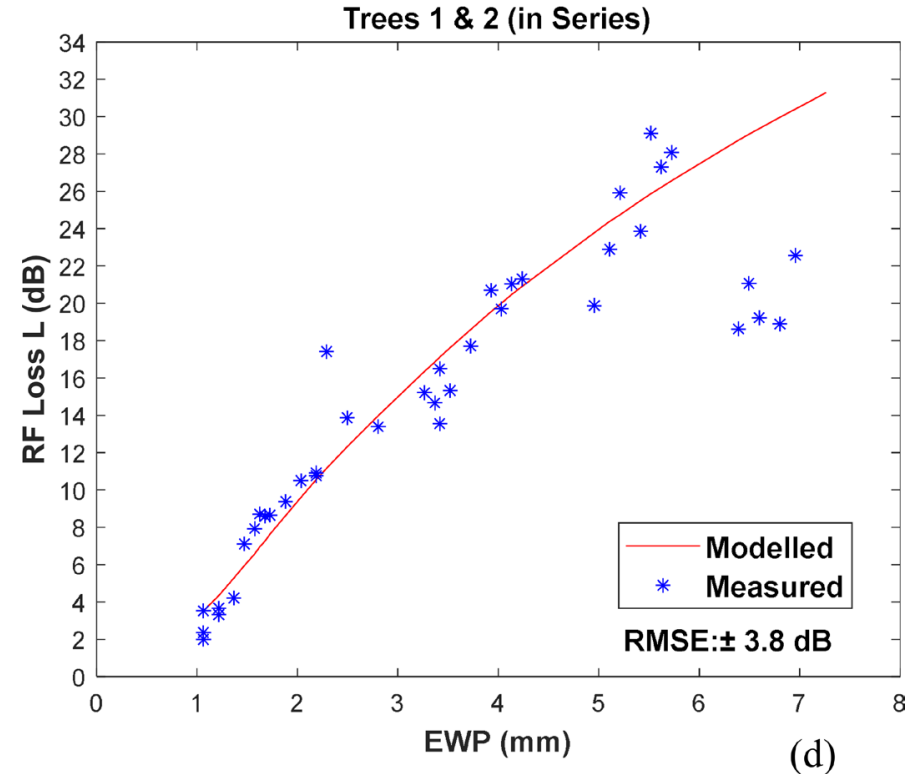

Figure 8. Plots of measured and modelled (Equation (13) with additional water content of $13 \%)$ RF loss (dB) as functions of EWP (mm) for Eucalyptus blakelyi trees ((a) Tree 1, (b) Tree 2; (c) Bare tree; (d) Trees $1 \& 2$ in series).

in its leaves ranging from $10 \%-30 \%$ (dry-weight basis) with other grass species exhibiting similar ranges and sometimes higher. In this earlier work, however, the bound water content is measured from freshly-sourced leaves which were not subjected to further desiccation. Here the values would be influenced by external factors such as soil moisture content, etc. [41].

An empirical approach available in this work is to identify the value of bound water that would elevate the modelled data values in Figure 7 to the measured values, effectively considering the actual water content of our trees to be higher (by this additional, bound contribution). We identified this value by finding the minimum total variance between measured and modelled values. To this end, the residual water was varied from $1 \%$ to $25 \%$ in $0.1 \%$ increments. The best fit between the modelled and the measured values is achieved by assuming that the tree canopies contained an additional water content of $13 \%$ when dried to constant weight. Residual water of this order of magnitude is plausible when compared against measurements of other leaf types [41]. Of course, what remains unclear is whether or not the "unfreezable" and "freezable" components of water identified by Whitman and others [38] [39] [40] is accessible through the leaf desiccation process in this work (or not) and whether the bound component is a contributor to the RF losses observed in this work.

Nevertheless, and with the new adjustment in the dry weight, the offset between the modelled and measured data collapses (refer Figure 8), reducing the RMSE by $31 \%$ - $42 \%$ compared to the unadjusted model.

\section{Conclusions}

A plane wave model, including an estimation of the bound water content of tree 
canopies, was developed to calculate the RF loss through eucalyptus tree canopy as a function of EWP at a frequency of $2.4 \mathrm{GHz}$. There was a positive non-linear relationship between RF loss in $\mathrm{dB}$ and the water content of the tree when the latter is expressed as EWP in mm. When the model was adjusted for additional water content of $13 \%$, EWP was found to explain $66 \%$ and $90 \%$ of the variance in the observed RF loss for single tree canopies with leaves and single tree without leaves respectively. It was also found to explain $75 \%$ of the variance when two trees with leaves were positioned in series.

The model developed in this research is compared against eucalyptus leaves and trees of some species. To generalize this model for wide range of tree types, the model needs to be compared against experiments acquired using other types of trees and other species of Eucalyptus. Verification of the model could also be done by using other parts of the tree as the lossy medium.

\section{Acknowledgements}

The first author acknowledges receipt of a Tuition Fee-Wavier Scholarship from the University of New England. One of us (DWL) acknowledges the support of Food Agility CRC Ltd, funded under the Commonwealth Government CRC Program. The CRC Program supports industry-led collaborations between industry, researchers, and the community. All authors gratefully acknowledge the contribution of Derek Schneider and Antony McKinnon from UNE for their help in setting up the experiment, and Prof. Jeremy Bruhl from UNE for helping us identify the eucalyptus species used for the experiment.

\section{Conflicts of Interest}

The authors declare no conflicts of interest regarding the publication of this paper.

\section{References}

[1] Boland, D.J., et al. (2006) Forest Trees of Australia. 5th Edition, Victoria CSIRO Publishing, Victoria.

[2] Matusick, G., Ruthrof, K., Brouwers, N., Dell, B. and Hardy, G. (2013) Sudden Forest Canopy Collapse Corresponding with Extreme Drought and Heat in a Mediterranean-Type Eucalypt Forest in Southwestern Australia. European Journal of Forest Research, 132, 497-510. https://doi.org/10.1007/s10342-013-0690-5

[3] ABARES (2018) Australia's State of the Forests Report. Department of Agriculture and Water Resources, Australia.

[4] Allen, C.D., et al. (2010) A Global Overview of Drought and Heat-Induced Tree Mortality Reveals Emerging Climate Change Risks for Forests. Forest Ecology and Management, 259, 660-684. https://doi.org/10.1016/j.foreco.2009.09.001

[5] Belyazid, S. and Giuliana, Z. (2019) Water Limitation Can Negate the Effect of Higher Temperatures on Forest Carbon Sequestration. European Journal of Forest Research, 138, 287-297. https://doi.org/10.1007/s10342-019-01168-4

[6] Kirkham, M.B. (2016) Elevated Carbon Dioxide: Impacts on Soil and Plant Water Relations. CRC Press, Boca Raton. https://doi.org/10.1201/b10812 
[7] Osakabe, Y., Osakabe, K., Shinozaki, K. and Tran, L.S. (2014) Response of Plants to Water Stress. Frontiers in Plant Science, 5, 86. https://doi.org/10.3389/fpls.2014.00086

[8] Pallardy, S., Pereira, J. and Parker, W. (1991) Measuring the State of Water in Tree Systems. In: Lassoie, J.P. and Hinckley, T.M., Eds., Techniques and Approaches in Forest Tree Ecophysiology, CRC Press, Boca Raton, 28-76.

[9] Pereira, J. and Pallardy, S. (1989) Water Stress Limitations to Tree Productivity. In: Biomass Production by Fast-Growing Trees, Springer, Berlin, 37-56. https://doi.org/10.1007/978-94-009-2348-5_3

[10] Zhou, J., Zhou, J., Ye, H., Ali, M.L., Nguyen, H.T. and Chen, P. (2020) Classification of Soybean Leaf Wilting Due to Drought Stress Using UAV-Based Imagery. Computers and Electronics in Agriculture, 175, Article ID: 105576. https://doi.org/10.1016/j.compag.2020.105576

[11] Resh, H.M. (2015) Signs of Plant Nutritional and Physiological Disorders and Their Remedies. In: Hydroponics for the Home Grower, CRC Press, Boca Raton, 76-89. https://doi.org/10.1201/b18069-16

[12] Peñuelas, J., Filella, I., Serrano, L. and Save, R. (1996) Cell Wall Elasticity and Water Index $($ R970 nm/R900 nm) in Wheat under Different Nitrogen Availabilities. International Journal of Remote Sensing, 17, 373-382. https://doi.org/10.1080/01431169608949012

[13] Peñuelas, J., Filella, I., Biel, C., Serrano, L. and Save, R. (1993) The Reflectance at the 950-970 nm Region as an Indicator of Plant Water Status. International Journal of Remote Sensing, 14, 1887-1905. https://doi.org/10.1080/01431169308954010

[14] Datt, B. (1999) Remote Sensing of Water Content in Eucalyptus Leaves. Australian Journal of Botany, 47, 909-923. https://doi.org/10.1071/BT98042

[15] LaGrone, A.H. (1960) Forecasting Television Service Fields. Proceedings of the IRE, 48, 1009-1015. https://doi.org/10.1109/JRPROC.1960.287501

[16] Balachander, D., Rao, T.R. and Mahesh, G. (2013) RF Propagation Experiments in Agricultural Fields and Gardens for Wireless Sensor Communications. Progress in Electromagnetics Research C, 39, 103-118. https://doi.org/10.2528/PIERC13030710

[17] Hristos, T.A., et al. (2014) A Computational Model for Path Loss in Wireless Sensor Networks in Orchard Environments. Sensors, 14, 5118-5135. https://doi.org/10.3390/s140305118

[18] Weissberger, M.A. (1982) An Initial Critical Summary of Models for Predicting the Attenuation of Radio Waves by Trees.

[19] Seville, A. and Craig, K. (1995) Semi-Empirical Model for Millimetre-Wave Vegetation Attenuation Rates. Electronics Letters, 31, 1507-1508. https://doi.org/10.1049/el:19951000

[20] Al-Nuaimi, M.O. and Stephens, R.B.L. (1998) Measurements and Prediction Model Optimisation for Signal Attenuation in Vegetation Media at Centimetre Wave Frequencies. IEE Proceedings-Microwaves, Antennas and Propagation, 145, 201-206. https://doi.org/10.1049/ip-map:19981883

[21] Adegoke, A.S. (2014) Measurement of Propagation Loss in Trees at SHF Frequencies. Ph.D. Thesis, Department of Engineering, University of Leicester, Leicester.

[22] Guo, X.-M. and Zhao, C. (2014) Propagation Model for $2.4 \mathrm{GHz}$ Wireless Sensor Network in Four-Year-Old Young Apple Orchard. International Journal of Agricultural and Biological Engineering, 7, 47-53.

[23] Rogers, N.C., et al. (2002) A Generic Model of 1-60 GHz Radio Propagation through 
Vegetation-Final Report. Radio Agency, UK.

[24] Jiang, S. and Georgakopoulos, S. (2011) Electromagnetic Wave Propagation into Fresh Water. Journal of Electromagnetic Analysis and Applications, 3, 261-266.

https://doi.org/10.4236/jemaa.2011.37042

[25] Da Silva, M.J. (2008) Impedance Sensors for Fast Multiphase Flow Measurement and Imaging. Ph.D. Thesis, Electrical and Computer Engineering Department, Technische Universität Dresden, Dresden.

[26] Lunkenheimer, P., et al. (2017) Electromagnetic-Radiation Absorption by Water. Physical Review E, 96, Article ID: 062607. https://doi.org/10.1103/PhysRevE.96.062607

[27] Le Vine, D.M. and Karam, M.A. (1996) Dependence of Attenuation in a Vegetation Canopy on Frequency and Plant Water Content. IEEE Transactions on Geoscience and Remote Sensing, 34, 1090-1096. https://doi.org/10.1109/36.536525

[28] Nakajima, I., Ohyama, F., Juzoji, H. and Ta, M. (2019) Developing a Scanner for Assessing Foliage Moisture. Journal of Multimedia Information System, 6, 155-164. https://doi.org/10.33851/JMIS.2019.6.3.155

[29] Shmulsky, R. and Jones, P.D. (2011) Wood and Water. In: Forest Products and Wood Science: An Introduction, 6th Edition, John Wiley \& Sons, Hoboken, 141-174. https://doi.org/10.1002/9780470960035.ch7

[30] Hasted, J.B. (1973) Aqueous Dielectrics. Chapman and Hall Ltd., London.

[31] Yang, W. and Siebenmorgen, T. (2003) Drying Theory. In: Encyclopedia of Agricultural, Food, and Biological Engineering, Marcel Dekker, New York, 231-241.

[32] Ulaby, F.T. and El-Rayes, M.A. (1987) Microwave Dielectric Spectrum of Vegetation Part II: Dual-Dispersion Model. IEEE Transactions on Geoscience and Remote Sensing, GE-25, 550-557. https://doi.org/10.1109/TGRS.1987.289833

[33] Peden, S., Bradbury, R.C., Lamb, D.W. and Hedley, M. (2021) A Model for RF Loss through Vegetation with Varying Water Content. Journal of Electromagnetic Analysis and Applications, 13, 41-56. https://doi.org/10.4236/jemaa.2021.133003

[34] Frickey, D.A. (1994) Conversions between S, Z, Y, H, ABCD, and T Parameters Which Are Valid for Complex Source and Load Impedances. IEEE Transactions on Microwave Theory and Techniques, 42, 205-211. https://doi.org/10.1109/22.275248

[35] Balanis, C.A. (2012) Advanced Engineering Electromagnetics. John Wiley \& Sons, Hoboken.

[36] Ulaby, F.T., Moore, R.K. and Fung, A.K. (1986) Microwave Remote Sensing: Active and Passive. Volume 3 From Theory to Applications. Artech House, Norwood.

[37] El-Rayes, M.A. (1987) The Measurement and Modeling of the Dielectric Behavior of Vegetation Materials in the Microwave Region (0.5-20.4 GHZ). Ph.D. Thesis, Department of Electrical and Computer Engineering, University of Kansas, Lawrence.

[38] Robinson, W. (1931) Free and Bound Water Determinations by the Heat of Fusion of Ice Method. Journal of Biological Chemistry, 92, 699-709. https://doi.org/10.1016/S0021-9258(17)32613-3

[39] Sayre, J.D. (1932) Methods of Determining Bound Water in Plant Tissue. Journal of Agricultural Research, 44, 669-688.

[40] Stark, A.L. (1932) An Apparatus and Method for Determining Bound Water in Plant Tissue. Proceedings of the American Society for Horticultural Science, 29, 384-388.

[41] Whitman, W.C. (1941) Seasonal Changes in Bound Water Content of Some Prairie Grasses. Botanical Gazette, 103, 38-63. https://doi.org/10.1086/335024 